\title{
PISA 2012: Inmigración y etapa de llegada al Sistema Educativo Español
}

\author{
PISA 2012: Age at Immigration and the Spanish Education System
}

\author{
Esperanza Bausela Herreras \\ Universidad Pública de Navarra, España
}

\begin{abstract}
Resumen
Introducción. Las evidencias empíricas muestran que hay diferencias en el rendimiento entre escolares inmigrantes que puede ser atribuido a diferentes variables, entre ellas la etapa de llegada al Sistema Educativo Español. Objetivo. Analizar el riesgo y/o probabilidad que escolares inmigrantes de la muestra española tienen de tener bajo rendimiento en las competencias evaluadas en PISA 2012, considerando la etapa de llegada al Sistema Educativo como variable predictora del riesgo. Metodología. No experimental o ex-post facto, diseño comparativo - causal. Análisis de datos. Análisis de regresión logística binaria. Resultados. En relación a la etapa de llegada al Sistema Educativo Español, es en la etapa de Educación Infantil donde se registra mayor riesgo de bajo de rendimiento en comparación con Educación Infantil: Lectura, Matemáticas y Ciencias. Respecto a las competencias, es en la competencia matemática donde se registra mayor riesgo en comparación con el resto de competencias evaluadas (Lectura y Ciencias). Conclusiones. El riesgo de tener bajo rendimiento varía según la etapa de llegada al Sistema Educativo. La llegada de escolares inmigrantes en Educación Infantil es un factor de riesgo. Es necesario desarrollar políticas educativas (programas lingüísticos, programas de acogida, por ejemplo) que minimicen el impacto y reduzcan el riesgo con el abandono y fracaso escolar en etapas posteriores.
\end{abstract}

Palabras clave: competencia, educación infantil, edad de llegada, inmigración, educación secundaria, Sistema Educativo Español, PISA 2012

\begin{abstract}
Introduction. Immigrants arriving at school at younger ages have lower educational achievement than those who arrive at adolescence. Aims. Analyze risk and / or probability of immigrant students having low performance in skills assessed in PISA 2012, considering their age of arrival into the Education System. Methodology. Non- experimental or ex post facto, comparative design - causal. Data Analysis: Binary logistic regression analysis. Outcomes. In relation to the age at entrance into the Spanish Education System, the stage of Early Childhood Education increases the risk of a diminished performance in Reading, Mathematics and Science. With respect to PISA 2012 skills assessment, it varies in stages: Kindergarten, Primary Education y Secondary Education. Conclusions. The risk of low performance varies with the educational stage at arrival. The arrival of immigrant students at Primary Education is a risk factor. We need to develop educational policies (e.g., language programs, host programs) that minimize the impact and reduce the risk of subsequent abandonment.

Keywords: Competence, Age at Immigration, High School, Spanish Education System, PISA 2012.

Esperanza Bausela Herreras, Área de Psicología Evolutiva y de la Educación, Departamento de Psicología y Pedagogía, Universidad Pública de Navarra (UPNA), España.

La correspondencia en relación con este artículo se dirige a Esperanza Bausela Herreras, Universidad Pública de Navarra (UPNA). Dirección electronica: esperanza.bausela@unavarra.es
\end{abstract}




\section{Introducción}

Los movimientos migratorios representan un tema de actual importancia social y, en concreto, en la política educativa. A pesar de la importancia de esta cuestión y la investigación respectiva, se desconoce aún sobre los factores que predicen el rendimiento de los escolares inmigrantes en comparación con sus compañeros nativos (ver Gould, Lavy, \& Paserman, 2009).

Son diversas las evidencias empíricas que muestran las diferencias en el rendimiento entre nativos versus inmigrantes (ver Calero \& Escardíbul, 2013; Cebolla-Boado, 2011, 2012; Dustmann, Frattini, \& Lanzara, 2011). Si nos centramos en escolares inmigrantes, que han llegado en la segunda mitad del siglo XX, Heat y Brinbaum (2007) enfatizan que la desigualdad y los logros varían considerablemente entre las comunidades étnicas, también dentro de las comunidades en relación con los países de acogida y las olas migratorias. Dustmann et al. (2011) mostraron que, en promedio, los escolares inmigrantes en la mayoría de los países europeos obtienen puntuaciones estadística y significativamente más bajas en las pruebas PISA que los escolares nativos. No obstante, hay excepciones. Así, en países como Irlanda, los escolares nativos obtienen puntuaciones más bajas. Estos datos varían en la segunda generación de pertenencia y respecto al número de progenitores inmigrantes.

Es difícil conocer cuáles elementos permiten explicar si las diferencias en el rendimiento entre inmigrantes versus nativos se debe a factores exclusivamente biológicos, a los relacionados con su entorno (social o cultural), a fenómenos de socialización o a la interacción entre ellos, tal como postula el enfoque de los sistemas ecológicos (Brofrenbrenner, 1976).

Los escolares inmigrantes se enfrentan a enormes desafíos cuando se incorporan al sistema educativo del país de acogida (ver Organisation for Economic Co-operation and Development [OECD], 2015) y estos desafíos varían en función de la edad y la etapa de llegada, entre otras variables. Por lo tanto, la incorporación tardía al sistema educativo del país receptor es una de las variables que se ha estudiado asociada con el rendimiento de los escolares inmigrantes (Böhlmark, 2008; Rahona \& Morales, 2013; Stiefel, Schwartz, \& Conger, 2010). No obstante, esta variable no tiene siempre el mismo impacto. Van Ours y Veenman (2006) señalan que, si la incorporación se produce a edades tempranas, la brecha en el rendimiento entre escolares inmigrantes versus escolares nativos se reduce en comparación si la incorporación se realiza tardíamente. Pereda, de Prada y Actis (1999) establecen, incluso, una edad -los ocho años- para determinar el pronóstico, porque a esta edad el ajuste y la adaptación son más leves y se agrava a partir de los doce años. Böhlmark (2008) sitúa los nueve años como la edad crítica de llegada, por encima de ella, considera que hay un fuerte riesgo de impacto negativo. Schaafsma y Sweetman (2001) -siguiendo la misma dirección que los autores anteriores- manifiestan que los escolares que llegan al final de la educación secundaria cuentan con tiempo, apenas, para prepararse y poder acceder a niveles superiores con los conocimientos básicos. No ocurre lo mismo para escolares inmigrantes que acceden a estudios de educación superior tras superar exhaustivos procesos de selección.

Porello,podemos plantearnos losiguiente: ¿existeunperíodooetapa críticoqueaumentaelriesgoyla probabilidaddetenerbajorendimiento en los escolaresinmigrantes?Como objetivodeesteestudioestáanalizarelriesgoy/oprobabilidaddequeescolaresinmigrantes(enmuestraespañola)tiendanaobtenerbajorendimientoenlastrescompetencias queevalúaPISA2012(Lectura,Matemáticas yCiencias)considerandolaetapa dellegadaalsistemaeducativoespañol. 


\section{Método}

El método empleado es no experimental o ex post facto y con diseño comparativo-causal. Al no haber manipulacióndevariables, solo se seleccionan losparticipantesy seagrupan en funciónde sus características; por ejemplo, condición de escolares en función de su etapa de llegada al sistema educativo español.

\section{Participantes}

La muestra generadora de datos está constituida por 25313 jóvenes españoles de ambos sexos, de 15 años, que pertenecen a 902 centros educativos distribuidos en todo el territorio nacional.

La base de datos utilizada fue la "base de datos PISA2012 española”, extraída de la propia página del Ministerio de Educación Cultura y Deporte (http://www.mecd.gob.es/inee/Bases-de-datos.html., Base de datos del informe PISA 2012, archivo alumnos [SPSS], archivo .zip) (fecha de consulta $1^{\circ}$ de abril de 2017).

De esta muestra total de España, 2694 escolares, escogidos para la presente investigación, son inmigrantes y pertenecen a 42 centros, que han ido llegado desde los 0 meses de vida hasta los 16 años de vida. A los cinco años cuando se registra el porcentaje más alto de escolares inmigrantes, 272, que representan el $10.10 \%$ y los 16 años registra el porcentaje más bajo, 9, que constituye el $0.33 \%$.

Los escolares inmigrantes han sido asignados a tres grupos en función del nivel del rendimiento en las tres competencias de PISA 2012 (alto, medio y bajo) y su etapa de llegada al sistema educativo español (educación infantil, educación primaria, educación secundaria obligatoria y bachillerato).

Los estudiantes fueron seleccionados aleatoriamente a partir de una muestra de escuelas públicas y privadas en función de su edad (entre 15 años y tres meses y 16 años y dos meses al principio de la evaluación) y no del grado escolar en el que se encuentran. Se seleccionaron, primero, los centros y luego los estudiantes, de forma aleatoria, que cumplieran con los criterios de inclusión en el estudio: los escolares deben tener 15 años cumplidos y, al menos, seis años de escolarización. Respecto a los criterios de exclusión, no pueden participar estudiantes con discapacidad intelectual o física y deben tener un dominio limitado de la lengua de enseñanza (menos de un año escolarizado en la lengua de la prueba que es la lengua de enseñanza).

En la tabla 1 se presenta una distribución (frecuencia y porcentaje) de escolares inmigrantes en función de la edad de llegada (años) (variable cuantitativa -discreta).

La edad de llegada, variable continua, ha sido agrupada en consonancia con las etapas educativas propias del sistema educativo español. Se ha trasformado en una variable cualitativa politómica, así: (i) de 0 a 6 años corresponde a educación infantil, (ii) de seis a 12 años en educación primaria, (iii), de 14 a 16 años equivale a educación secundaria obligatoria y (iv) de 16 a18 años corresponde a bachillerato.

Respecto, a la etapa de llegada al sistema educativo español, el 49.55\% (casi el 50\% de los inmigrantes) llegan a lo largo de la etapa de educación primaria (etapa obligatoria), mientras que el $0.33 \%$ en bachillerato (etapa no obligatoria) (variable cualitativa politómica). 
E. BAUSELA

Tabla 1

Edad de llegada al país de acogida (años) de los escolares inmigrantes en PISA 2012 (frecuencia y porcentaje)

\begin{tabular}{ccc}
\hline Edad de llegada al país de acogida (años) & Frecuencia & Porcentaje \\
\hline 0 & 64 & 2.38 \\
1 & 54 & 2.00 \\
2 & 124 & 4.60 \\
3 & 149 & 5.53 \\
4 & 215 & 7.98 \\
5 & 272 & 10.10 \\
6 & 244 & 9.06 \\
7 & 224 & 8.31 \\
8 & 197 & 7.31 \\
9 & 202 & 7.50 \\
10 & 253 & 9.39 \\
11 & 215 & 7.98 \\
12 & 191 & 7.09 \\
13 & 115 & 4.27 \\
14 & 111 & 4.12 \\
15 & 55 & 2.04 \\
16 & 9 & 0.33 \\
Total & 2694 & 100 \\
\hline
\end{tabular}

En la tabla 2 se presenta una distribución (frecuencia y porcentaje) de escolares inmigrantes en función de la etapa de llegada al sistema educativo español.

Tabla 2

Etapa de llegada al sistema educativo español de escolares inmigrantes en PISA 2012 (frecuencia y porcentaje)

\begin{tabular}{lcc}
\hline Etapa del sistema educativo español & Frecuencia & Porcentaje \\
\hline Educación infantil & 878 & 32.59 \\
Educación primaria & 1335 & 49.55 \\
Educación secundaria & 472 & 17.52 \\
Bachillerato & 9 & 0.33 \\
Total & 2694 & 100 \\
\hline
\end{tabular}


PISA 2012: InMigración y ETAPA DE LLEGAdA AL Sistema Educativo Español

En el Anexo I y II se presenta la distribución de los participantes en los diferentes centros escolares participantes en función de la edad de llegada y la etapa educativa.

Respecto al rendimiento en las competencias evaluadas en PISA (Lectura, Matemáticas y Ciencias), se presenta la frecuencia y el porcentaje en relación con la naturaleza misma de dichos aspectos (ver tabla 3).

\section{Tabla 3}

Descriptivos del rendimiento en las competencias evaluadas en PISA 2012 (lectura, Matemáticas y Ciencias)

\begin{tabular}{|c|c|c|}
\hline Competencia/Nivel & Frecuencia & Porcentaje \\
\hline \multicolumn{3}{|l|}{ Lectura } \\
\hline Alto & 41 & 1.52 \\
\hline Bajo & 1711 & 63.51 \\
\hline Medio & 942 & 34.97 \\
\hline \multicolumn{3}{|l|}{ Matemáticas } \\
\hline Alto & 6 & 0.22 \\
\hline Bajo & 1899 & 70.49 \\
\hline Medio & 789 & 29.29 \\
\hline \multicolumn{3}{|l|}{ Ciencias } \\
\hline Alto & 50 & 1.86 \\
\hline Bajo & 1720 & 63.85 \\
\hline Medio & 924 & 34.30 \\
\hline
\end{tabular}

\section{Hipótesis y variables de investigación}

\section{Hipótesis de investigación}

Las hipótesis de investigación que guían este estudio son las siguientes:

- Hipótesis nula: Los escolares inmigrantes, que aceden en las últimas etapas del sistema educativo español (educación secundaria obligatoria y bachillerato), no corren más riesgo de mantener bajo rendimiento en las competencias evaluadas en PISA 2012 (Lectura, Matemáticas y Ciencias) en comparación con los escolares inmigrantes que acceden en las primeras etapas del sistema educativo español (educación infantil y educación primaria).

- Hipótesis alternativa: Los escolares inmigrantes, que aceden en las últimas etapas del sistema educativo español (educación secundaria obligatoria y bachillerato), poseen más riesgo de mantener bajo 
rendimiento en las competencias evaluadas en PISA 2012 (Lectura, Matemáticas y Ciencias) en comparación con los escolares inmigrantes que acceden en las primeras etapas del sistema educativo español (educación infantil y educación primaria).

\section{Variables de investigación}

Según el papel que desempeñan en la investigación las variables son las siguientes:

\section{Variable dependiente}

Para el presente estudio, la variable dependiente corresponde al rendimiento obtenido en las competencias que se evalúan en PISA 2012 (Lectura, Matemáticas y Ciencias) (variable cuantitativa continua) es operacionalizado en relación con su rendimiento en una variable cualitativa politómica: alto, medio y bajo en dichas competencias, de acuerdo con los siguientes criterios (Ministerio de Educación Cultura y Deporte [MECD] \& Organización para la Cooperación Y Desarrollo Economico [OCDE], 2013a; Ministerio de Educación Cultura y Deporte [MECD] \& Organización para la Cooperación Y Desarrollo Economico [OCDE], 2013b): (i) Así, un estudiante de nivel de competencia alto, es aquel que seguramente completará con éxito los ítems I-V y probablemente VI, también. (ii) Un estudiante con un nivel de competencia medio, seguramente completará con éxito los ítems I y II, probablemente el ítem III, pero no los ítems V y VI y seguramente tampoco el ítem IV. (iii) El estudiante con nivel de competencia bajo es probable que no complete con éxito los ítems I, II-IV..

En este estudio concretamente, además, hemos trasformado dicha variable cualitativa politómica en dicotómica. De esta forma, el bajo rendimiento se identifica y es equivalente al nivel bajo en las tres competencias que se evalúan en PISA 2012 (Lectura, Matemáticas y Ciencias): Si bajo rendimiento que equivale al nivel bajo en dichas competencias (Lectura, Matemáticas y Ciencias) y no bajo rendimiento que equivale al resto de niveles (medio y alto).

\section{Variable independiente}

La variable independiente hace referencia a la etapa de llegada al sistema educativo español, operacionalizada en consonancia con dicho sistema (Ministerio de Educación, Cultura y Deporte, s.f): educación infantil, educación primaria, educación secundaria y bachillerato. En concreto, la etapa de educación infantil en el sistema de educación español, como el propio Ministerio de Educación, Cultura y Deporte señala, es la etapa educativa que atiende a niñas y niños desde el nacimiento hasta los seis años con la finalidad de contribuir a su desarrollo físico, afectivo, social e intelectual. Se ordena en dos ciclos: (i) El primero comprende hasta los 3 años (no es gratuito, por ello se desarrollan iniciativas como el Programa Educa 3 con el objetivo de fomentan la creación de nuevas plazas educativas para niños de menos de tres años y (ii) el segundo, que es gratuito, incluye edades desde los tres a los seis años.

La educación primaria tiene carácter obligatorio y gratuito. Comprende seis cursos académicos, que se seguirán ordinariamente entre los seis y los doce años de edad. Con carácter general, el alumnado se incorporará al primer curso de la educación primaria en el año natural en el que cumplan seis años.

La educación secundaria obligatoria (ESO) es una etapa educativa obligatoria y gratuita que completa la educación básica. Consta de cuatro cursos académicos que se realizan ordinariamente entre los 
PISA 2012: InMigración y ETAPA DE LLEGAdA AL Sistema EduCATIVo EsPañol

12 y los 16 años de edad. No obstante, los alumnos tendrán derecho a permanecer en régimen ordinario cursando la enseñanza básica hasta los dieciocho años de edad.

El bachillerato forma parte de la educación secundaria posobligatoria y, por lo tanto, posee carácter voluntario. Comprende dos cursos académicos, que se realizan ordinariamente entre los 16 y 18 años de edad.

\section{Análisis de datos}

Los datos fueron sometidos inicialmente a análisis inferenciales: bivariados (estadístico de asociación Chi-Cuadrado) y multivariados (regresión logística binaria) que seguidamente comentamos.

Se analiza la asociación entre las variables dependientes (rendimiento en las tres competencias) y la variable independiente principal (etapa de llegada al sistema educativo español) con Chi-cuadrado de Pearson, que nos permite contrastar la hipótesis que el nivel de bajo rendimiento es igual en todas las etapas del sistema educativo. Si la hipótesis nula fuera cierta, se deberían encontrar porcentajes similares en el rendimiento entre grupos de escolares inmigrantes que acceden al sistema educativo español en diferentes etapas: educación infantil, educación primaria, educación secundaria obligatoria y bachillerato.

Se aplicó la técnica de regresión logística binomial (modelo logit) (Logistic Probability Unit) (método introducir) (es decir, entrando cada vez en el modelo una de las variables independientes o de control) con la finalidad de predecir la ocurrencia de bajo rendimiento (sí/no) en función de la etapa de llegada al sistema educativo español. Estimamos que la técnica más adecuada para responder al objetivo de la investigación y que se ajusta a la naturaleza de las variables dependientes (nominal, bivariada), es el modelo logit (Logistic Probability Unit) (método introducir).

El Odds Ratio (OR) expresa si la probabilidad de ocurrencia de un evento, acontecimiento o enfermedad, por ejemplo: caso/no caso difiere o no en distintos grupos, por lo general catalogados de alto o bajo riesgo, pero debido a que no posee límites claros es difícil interpretarlo. Su información es fundamentalmente descriptiva, aunque si su intervalo de confianza (IC) no incluye al 1 se concluye que la asociación es estadísticamente significativa; es decir, que la cantidad de casos que posee el grupo de alto riesgo resulta significativamente más grande que la cantidad de casos que ubicados en el grupo de bajo riesgo. Así, exceptuando en la competencia lectora, cuando los escolares llegan en la etapa de Bachillerato, en todos los demás grupos analizados se observa a partir de la Odds Ratio que la cantidad de casos que posee el grupo de alto riesgo es significativamente más grande que la cantidad de casos que ubicados en el grupo de bajo riesgo.

La hipótesis de independencia de los residuos se ha realizado mediante el contraste de DurbinWatson, mediante un análisis de regresión lineal (predictores: constante; residuo normalizado; variable dependiente: probabilidad pronosticada).

El contraste $d$ Durbin - Watson es la prueba más frecuentemente empleada para detectar la presencia de autocorrelación en los modelos de regresión. Los valores obtenidos en las tres competencias son próximos a dos. Por ello, no se rechaza la hipótesis nula (se rechaza la hipótesis alternativa). Se podría afirmar que no existe autocorrelación. 
Los datos fueron analizados con el programa estadístico SPSS versión 24 (versión institucional). Se emplearon los módulos relacionados con estadísticos y regresión (logística binaria). Los gráficos son de elaboración propia utilizando el programa Excel de Microsoft.

\section{Resultados}

Se presentan, seguidamente, los resultados en relación con la asociación entre el nivel de competencia y la etapa de llegada al sistema educativo español y la predicción del rendimiento en función de la etapa de llegada al sistema educativo español por escolares inmigrantes.

\section{Asociación del nivel de competencia (alto, medio y bajo) en función de la etapa de llegada al Sistema Educativo Español por escolares inmigrantes.}

La etapa de llegada al sistema educativo español es una variable que se obtiene a partir de años de la edad de llagada al sistema educativo español (información disponible en la propia base de datos de PISA 2012), al igual que la condición de inmigrante.

Como se ha indicado en páginas precedentes, el nivel de competencia (alto, medio y bajo) se consideró en función de los niveles referenciados por (MECD y OCDE, 2013a; MEC y OCDE, 2013b). La distribución de la muestra en función de los niveles de competencia se presenta en la tabla 4. Los datos nos indican que: (i) El nivel alto en competencia lectora, matemática y científica se asocia con la llegada en la llegada en educación infantil; (ii) el nivel medio en competencia matemática y científica se asocia con la llegada en la etapa de educación primaria y baja competencia lectora con dicha etapa educativa (educación primaria); (iii) el nivel bajo en competencia lectora, matemática y científica se asocia con la llegada en la etapa de educación secundaria obligatoria y bachillerato. En la tabla 4 se presentan los niveles de rendimiento en las competencias (Lectura, Matemáticas y Ciencias) y puntos de corte en PISA 2012 (variable cualitativa politómica), así como el nivel de bajo rendimiento (variable cualitativa dicotómica).

Tabla 4

Niveles de rendimiento en las competencias (Lectura, Matemáticas y Ciencias) y puntos de corte en PISA 2012

\begin{tabular}{ccccc}
\hline \multicolumn{2}{c}{ Competencias PISA 2012 } & $\begin{array}{c}\text { Niveles de competencia } \\
\text { (politómica) }\end{array}$ & $\begin{array}{c}\text { Niveles de bajo } \\
\text { rendimiento (dicotómica) }\end{array}$ \\
\hline Lectura & Matemáticas & Ciencias & & SÍ \\
\hline$[262.0-480.2]$ & {$[357.7-482.4]$} & {$[334.9-484.1]$} & Bajo (Item I - Ítem II) & NO \\
{$[480.2-625.6]$} & {$[482.4-607.0]$} & {$[484.1-633.3]$} & Medio (Ítem III - Ítem IV) & NO \\
{$[625.6-698.3]$} & {$[607.0-669.3]$} & {$[633.3-707.9]$} & Alto (Ítem V - Ítem VI) & \\
\hline
\end{tabular}

Se obtiene una asociación estadísticamente significativa entre el nivel de competencia (alto, medio y bajo) en las tres competencias que se evalúan en PISA 2012 (lectora, matemática y científica) y la etapa de llegada al sistema educativo español de los escolares inmigrante a través del coeficiente de asociación Chi-cuadrado (ver tabla 5): Lectura $\left[\chi^{2}=69.759, g l=6, p<.001\right]$, Matemáticas $\left[\chi^{2}=68.468, g l=6, p\right.$ 
PISA 2012: InMigración y ETAPA DE LLEGAdA AL Sistema EduCATIVo EsPañol

$<.001]$, Ciencias $\left[\chi^{2}=68.880, g l=6, p<.001\right]$, como la significancia es menor que .05 se rechaza Ho, por lo que la proporción de escolares con diferentes niveles de competencia (alto, bajo y medio) es diferente entre escolares inmigrantes que llegaron en diferentes etapas educativas del sistema educativo español. Con 1 grado de libertad y un valor de confianza del 95\%, este valor es estadísticamente significativo en las tres competencias.

\section{Tabla 5}

Porcentaje, frecuencia y Chi-cuadrado del nivel de competencia (alto, medio y bajo) PISA 2012 en función de la etapa de llegada al sistema educativo español de escolares inmigrantes

\begin{tabular}{|c|c|c|c|c|c|c|}
\hline \multirow{3}{*}{$\begin{array}{l}\text { Competencia/ } \\
\text { niveles }\end{array}$} & \multicolumn{4}{|c|}{ Etapa de llegada del sistema educativo español } & \multirow{2}{*}{ Total } & $\chi^{2}$ \\
\hline & Ed. Infantil & $\begin{array}{c}\text { Ed. } \\
\text { Primaria }\end{array}$ & $\begin{array}{c}\text { Ed. Secundaria } \\
\text { Obligatoria }\end{array}$ & Bachillerato & & Valor \\
\hline & $\% n$ & $\% n$ & $\% n$ & $\% \quad n$ & $\% n$ & \\
\hline \multicolumn{7}{|l|}{ Lectura } \\
\hline Alto & $43.9(18)$ & $39.0(16)$ & $17.1(7)$ & $0(0)$ & $100(41)$ & \multirow[t]{3}{*}{$69.759^{\mathrm{a}}(6)(.001) * * *$} \\
\hline Bajo & $28.9(495)$ & $49.0(838)$ & $21.6(370)$ & $0.5(8)$ & $100(1711)$ & \\
\hline Medio & $38.7(365)$ & $51.1(481)$ & $10.1(95)$ & $0.1(1)$ & $100(942)$ & \\
\hline \multicolumn{7}{|l|}{ Matemáticas } \\
\hline Alto & $50(3)$ & $16.7(1)$ & $33.3(2)$ & $0(0)$ & $100(6)$ & \multirow[t]{3}{*}{$68.468^{\mathrm{b}}(6)(.001) * * *$} \\
\hline Bajo & $29.2(554)$ & $49.6(942)$ & $20.7(394)$ & $0.5(9)$ & $100(1899)$ & \\
\hline Medio & $40.7(321)$ & $49.7(392)$ & $9.6(76)$ & $0(0)$ & $100(789)$ & \\
\hline \multicolumn{7}{|l|}{ Ciencias } \\
\hline Alto & $50.0(25)$ & $40.0(20)$ & $10.0(5)$ & $0(0)$ & $100(50)$ & \multirow[t]{3}{*}{$68.880^{c}(6)(.001) * * *$} \\
\hline Bajo & $28.9(497)$ & $49.1(845)$ & $21.5(370)$ & $0.5(8)$ & $100(1720)$ & \\
\hline Medio & $38.5(356)$ & $50.9(470)$ & $10.5(97)$ & $0.1(1)$ & $100(924)$ & \\
\hline
\end{tabular}

Nota: Fuente: [Elaboración propia a partir de PISA 2012 (muestra española)].

a. 2 casillas $(16.7 \%)$ han esperado un recuento menor que 5. El recuento mínimo esperado es .14.

b. 5 casillas $(41.7 \%)$ han esperado un recuento menor que 5 . El recuento mínimo esperado es .02 .

c. 2 casillas $(16.7 \%)$ han esperado un recuento menor que 5 . El recuento mínimo esperado es .17 .

$* * * p<.001$.

\section{Asociación del bajo rendimiento en función de la etapa de llegada al sistema educativo español por escolares inmigrantes.}

Los escolares inmigrantes han sido asignados a dos grupos en función del nivel del rendimiento en el nivel bajo (que hemos identificado con bajo rendimiento) en las tres competencias de PISA 2012 (SÍ/ 
NO) y su etapa de llegada al sistema educativo español (educación infantil, educación primaria, educación secundaria obligatoria y bachillerato).

La distribución de la muestra en función del bajo rendimiento (nivel bajo) se presenta en la tabla 6. Los datos nos indican que el bajo nivel alto en competencia lectora, matemática y científica se asocia con la llegada en la llegada en educación primaria.

Tabla 6

Porcentaje, frecuencia y Chi-Cuadrado del bajo rendimiento (nivel bajo) en las tres competencias PISA 2012 en función de la etapa de llegada al sistema educativo español de escolares inmigrantes.

\begin{tabular}{|c|c|c|c|c|c|c|c|}
\hline \multirow{3}{*}{$\begin{array}{l}\text { Competencia/ } \\
\text { Bajo } \\
\text { rendimiento }\end{array}$} & \multicolumn{4}{|c|}{ Etapa de llegada del sistema educativo español } & \multirow{3}{*}{$\begin{array}{l}\text { Total } \\
\% \quad n\end{array}$} & \multicolumn{2}{|l|}{$\chi^{2}$} \\
\hline & Ed. Infantil & $\begin{array}{c}\text { Ed. } \\
\text { Primaria }\end{array}$ & $\begin{array}{c}\text { Ed. Secundaria } \\
\text { Obligatoria }\end{array}$ & Bachillerato & & Valor $\quad \mathrm{gl}$ & $p$ \\
\hline & $\% \quad n$ & $\% n$ & $\% \quad n$ & $\% \quad n$ & & & \\
\hline Lectura & & & & & & $67.18^{\mathrm{a}}(3)(.001)$ & $* * *$ \\
\hline SÍ & $28.9(495)$ & $49(838)$ & $21.6(370)$ & $0.5(8)$ & $100(1711)$ & & \\
\hline $\mathrm{NO}$ & $39(383)$ & $50.6(497)$ & $10.4(102)$ & $0.1(1)$ & $100(983)$ & & \\
\hline Matemáticas & & & & & & $65.09^{\mathrm{b}}(3)(.001)$ & $* * *$ \\
\hline SÍ & $29.2(554)$ & $49.6(942)$ & $20.7(394)$ & $9(0.5)$ & $100(1899)$ & & \\
\hline NO & $40.8(324)$ & $49.4(393)$ & $9.8(78)$ & $0(0)$ & $100(795)$ & & \\
\hline Ciencias & & & & & & $65.81^{\mathrm{c}}(3)(.001)$ & $* * *$ \\
\hline SÍ & 28.9 (497) & $49.1(845)$ & $21.5(370)$ & $0.5(8)$ & $100(1720)$ & & \\
\hline NO & $39.1(381)$ & $50.3(490)$ & $10.5(102)$ & $0.1(1)$ & $100(974)$ & & \\
\hline
\end{tabular}

Nota: Fuente: [Elaboración propia a partir de PISA 2012 (muestra española)].

a. 1 casillas $(12.5 \%)$ han esperado un recuento menor que 5. El recuento mínimo esperado es 3.28.

b. 1 casillas $(12.5 \%)$ han esperado un recuento menor que 5. El recuento mínimo esperado es 2.66.

c. 1 casillas $(12.5 \%)$ han esperado un recuento menor que 5. El recuento mínimo esperado es 3.25 .

$* * * p<.001$.

Se obtiene una asociación estadísticamente significativa entre el nivel bajo -identificado como bajo rendimiento en las tres competencias evaluadas en PISA 2012 (lectura, matemáticas y ciencias)- y la etapa de llegada al sistema educativo español de los escolares inmigrantes a través del coeficiente de 
asociación Chi-cuadrado (ver tabla 6). Lectura $\left[\chi^{2}=67.18, g l=3, p<.001\right]$, Matemáticas $\left[\chi^{2}=65.09, g l\right.$ $=3, p<.001]$, Ciencias $\left[\chi^{2}=65.81, g l=3, p<.001\right]$, como la significancia es menor que .05 se rechaza Ho, por lo que la proporción de escolares con bajo rendimiento es diferente entre escolares inmigrantes que llegaron en diferentes etapas educativas del sistema educativo español.

\section{Predicción del riesgo de bajo rendimiento en función de la etapa de llegada al sistema educativo español por escolares inmigrantes: regresión logística binaria}

Con el fin de predecir la ocurrencia de bajo rendimiento en función de la etapa de llegada al sistema educativo español en relación con las tres competencias evaluadas en PISA 2012 y considerando la naturaleza de las variables dependientes (nominal, bivariada), se optó por el modelo logit (Logistic Probability Unit) (método introducir).

Los resultados de $\beta$ y Odds Ratio (OR), equivalente a $\operatorname{Exp}(\beta)$ a un nivel del 95\% (límite inferior y superior) y el contraste d Durbin - Watson, se presentan en la tabla 7.

Respecto al valor que se incluye en la tabla 7, porcentaje de clasificación o también conocido de predicción, hace referencia a un formato estándar, caracterizado por el cruce de las frecuencias de las opciones de respuestas de Y observadas en la muestra ( 1 ocurrencia del evento: 0 no ocurrencia) con las predichas $\left(\mathrm{Y}^{\wedge}\right)$, a partir del modelo estimado. La eficacia predictiva, también, puede evaluarse de forma gráfica mediante la curva ROC.

La probabilidad de ser favorable, es decir, de tener bajo rendimiento en las competencias evaluadas en PISA 2012 (Lectura, Matemáticas y Ciencias) se ha calculado al aplicar la fórmula genérica (ecuación de regresión logística binaria) (ver Cea D’Ancona, 2011):

$$
\begin{array}{r}
\operatorname{Logit}(\mathrm{Y})=\log \left[\frac{\mathrm{P}(\mathrm{Y}=\text { bajo rendimiento })}{\mathrm{P}(\mathrm{Y}=\text { no bajo rendimiento })}\right] \\
=\alpha+\beta_{1} \mathrm{X}_{1}+\beta_{2} \mathrm{X}_{2}+\cdots \cdot+\beta_{\mathrm{K}} \mathrm{X}_{\mathrm{K}}
\end{array}
$$

Se observa que el riesgo de tener bajo rendimiento es: (i) En relación con las tres competencias, objeto de estudio, en Matemática se registra mayor riesgo de bajo rendimiento en comparación con el resto de las evaluadas (Lectura y Ciencias). (ii) En cuanto a las etapas de llegada al sistema educativo español, en la etapa de educación infantil y educación primaria se evidenció mayor riesgo de bajo de rendimiento.

En la figura 1 se representa el riesgo de bajo rendimiento de escolares inmigrantes en función de la etapa de llegada al sistema educativo español en las tres competencias de PISA2012 (Lectura, Matemáticas y Ciencias). (iii) En relación con la etapa de llegada al sistema educativo español, los datos indican que el riesgo de tener bajo rendimiento varía en las tres competencias. Así, en las competencias lectora y científica el riesgo es mayor en la etapa de educación infantil, mientras que en la competencia matemática el riesgo es igual en las tres primeras etapas del sistema educativo español (infantil, primaria y secundaria obligatoria). 
ก․

इ. कृ कृ

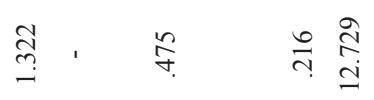

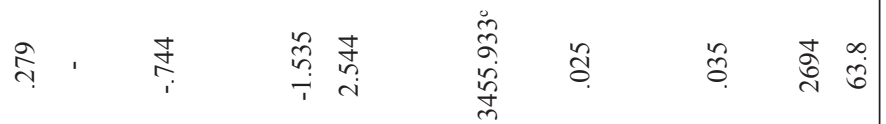

。용

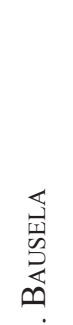

: สู่

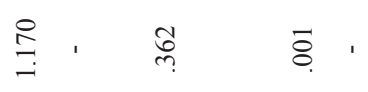

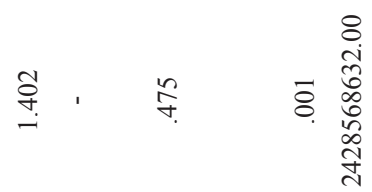

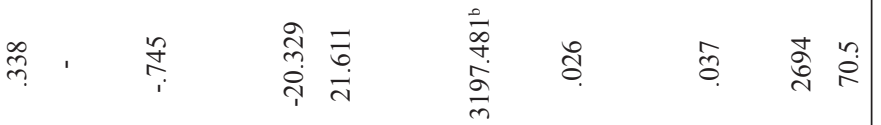

㿿。

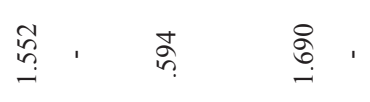

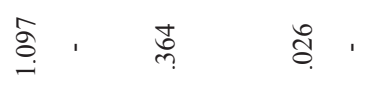

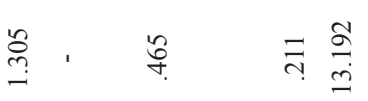

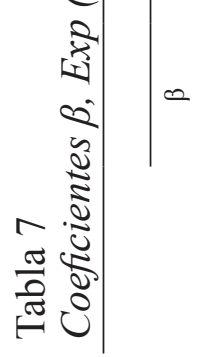




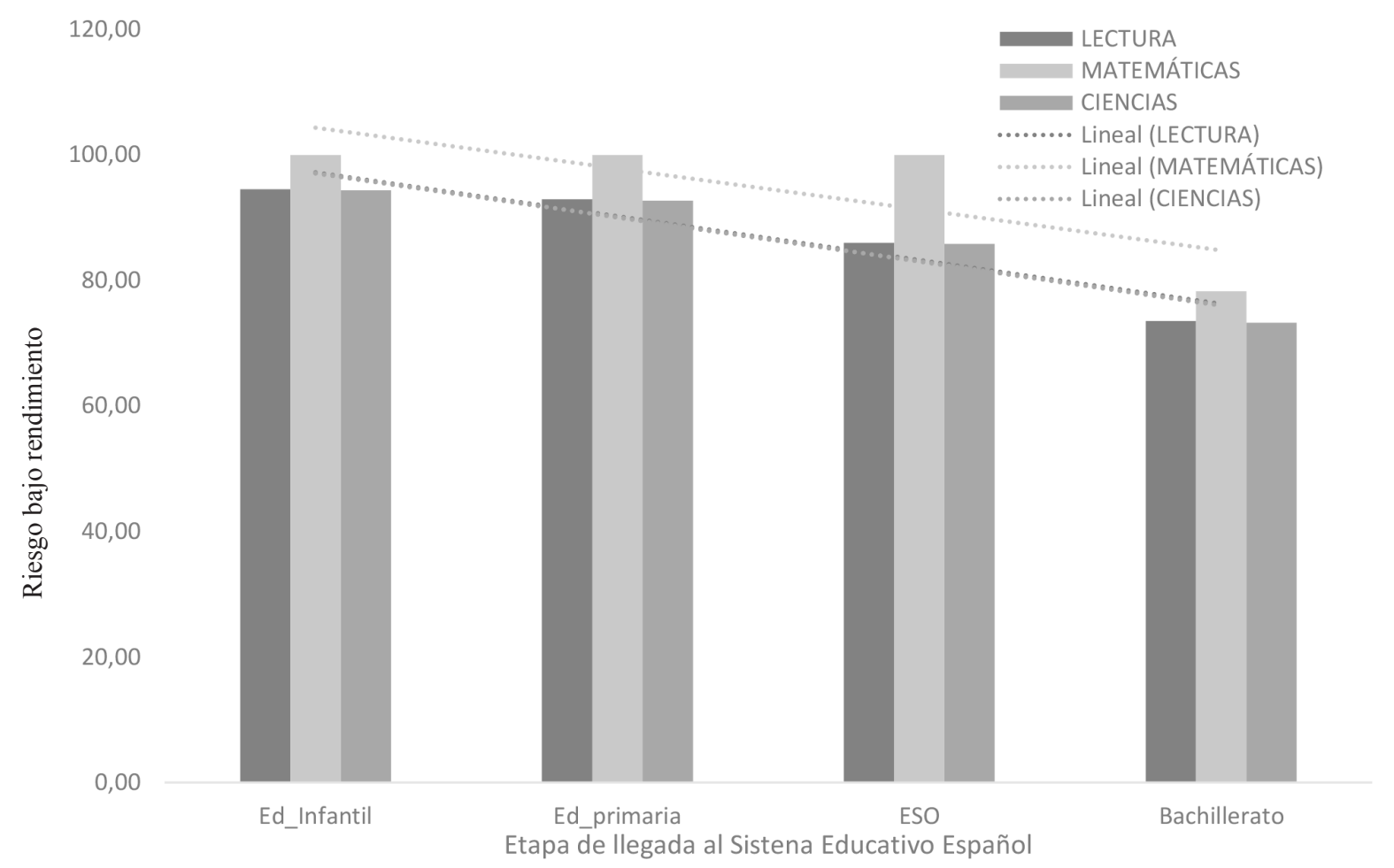

Figura 1. Riesgo de bajo rendimiento en escolares inmigrantes en función de la etapa de llegada al sistema educativo español en las tres competencias de PISA 2012 (riesgo y tendencia).

\section{Conclusiones y discusiones}

El objetivo del presente estudio era analizar, en escolares inmigrantes en función de la etapa de llegada al sistema educativo español, el riesgo de tener bajo rendimiento de. Como hipótesis de investigación, nos habíamos planteado que los sujetos de estudio, quienes acceden en las últimas etapas del sistema educativo español (educación secundaria obligatoria y bachillerato) poseen más riesgo de tener bajo rendimiento en las competencias evaluadas en PISA 2012 (Lectura, Matemáticas y Ciencias) en comparación con los escolares que acceden en las primeras etapas del sistema educativo español (educación infantil y educación primaria).

Los resultados de la base de datos de PISA 2012 (muestra española, submuestra de inmigrantes) confirman que el riesgo de tener bajo rendimiento varía en función de la etapa de llegada al sistema educativo en las tres competencias evaluadas en PISA 2012.

Los niveles de competencia curricular exigidos en educación infantil y educación secundaria son claramente diferentes. Así, en educación infantil el interés está en la adquisición de los prerrequisitos 
básicos para la adquisición de las habilidades instrumentales (lectura, escritura y cálculo) con un carácter eminentemente propedéutico y compensador de desigualdades; en educación secundaria el foco de interés está en la preparación al estudiante para el acceso al bachillerato, puesto que Llas exigencias académicas superiores requieren comprender y expresar con corrección, oralmente y por escrito, en la lengua castellana y, si la hubiere, en la lengua cooficial de la Comunidad Autónoma (ver Real Decreto 1630/2006, Real Decreto 126/2014, Real Decreto 1105/2014).

Los datos obtenidos muestran evidencias de que la incorporación más temprana al sistema educativo español incrementa significativamente el riesgo de tener bajo rendimiento en las tres competencias que son evaluadas en PISA 2012. Estos resultados nos llevan a rechazar la hipótesis de investigación que hace referencia a que la incorporación tardía incrementa el riesgo de tener bajo rendimiento.

Estos resultados están en la línea de los obtenidos por Pereda et al. (1999); Fernández Enguita, Mena y Riviére (2010), aunque es importante señalar que los resultados del presente estudio no se pueden comparar, porque solo se ha incluido la variable etapa de llegada al sistema educativo español. Debemos considerar que el rendimiento académico no se puede explicar con una sola variable.

Estos datos nos confirman la importancia de la identificación precoz de las necesidades de apoyo educativo que presentan los escolares inmigrantes para minimizar su impacto y disponer de estrategias de "acogida", incluyendo, por ejemplo, mecanismo para facilitar el acceso a la lengua sin perder su identidad y políticas educativas que contribuyan a fomentar las relaciones entre los diferentes sistemas educativos. Los estudios han analizado los contrastes en el rendimiento entre escolares nativos e inmigrantes y se centran en analizar las diferencias en función de variables socioeconómicas (Cebolla-Boado, 2011; Oliden, 2013), peso al nacer (Cebolla-Boado \& Salazar, 2016). No obstante, estas variables tienen diferente impacto en función de los países y los diferentes sistemas educativos. Por lo tanto, no es posible generalizar los resultados obtenidos en este estudio con el Sistema Educativo Español a países con otros Sistemas Educativos.

En esta dirección apuntan el Plan PROA (Programas de Refuerzo, Orientación y Apoyo), el cual se concibe como un proyecto de cooperación territorial entre el Ministerio de Educación y las Comunidades Autónomas. Pretende abordar las necesidades asociadas al entorno sociocultural del alumnado mediante un conjunto de programas de apoyo a los centros educativos: (i) Programa de acompañamiento escolar en primaria, (ii) Programa de acompañamiento escolar en secundaria, (iii) Programa de apoyo y refuerzo en educación secundaria (Comunidad de Madrid, s.f.).

El Plan PROA ofrece recursos a los centros educativos para que, junto a los demás actores de la educación, trabajen en una doble dirección: contribuir a debilitar los factores generadores de la desigualdad y garantizar la atención a los colectivos más vulnerables para mejorar su formación y prevenir los riesgos de exclusión social. Se persiguen tres objetivos estratégicos: Lograr el acceso a una educación de calidad para todos, enriquecer el entorno educativo e implicar a la comunidad local (Comunidad de Madrid, s.f.).

Este programa se puede vincular con la normativa vigente -Ley Orgánica 8/2013, de 3 de mayo, de Educación- que menciona explícitamente hacer efectivo el principio de igualdad en el ejercicio del derecho a la educación en correspondencia con las administraciones públicas a desarrollar acciones de 
PISA 2012: InMigración y ETAPA DE LLEGAdA AL Sistema Educativo Español

carácter compensatorio en relación con las personas, los grupos y los ámbitos territoriales que se encuentren en situaciones desfavorables y proveerán los recursos y los apoyos precisos para ello.

\section{Limitaciones y líneas de investigación futuras}

En este estudio nos hemos centrado en analizar el riesgo que conlleva para los escolares inmigrantes llegar en diferentes etapas educativas del sistema educativo español. Por ello, estimamos necesario, para un futuro, considerar cómo incide el riesgo de bajo rendimiento en función de otras variables relacionadas con el escuela (por ejemplo, concentración de escolares inmigrantes en centros educativos) (ver Carabaña, 2012) y con la propia familia (por ejemplo, estatus ocupacional de los progenitores) que pueden estar asociadas con el rendimiento en las competencias evaluadas en PISA 2012 y que cobran especial importancia en escolares inmigrantes (Ozel, Caglak \& Erdogan, 2013).

Puede ser, igualmente interesante, conocer la relación entre la inmigración, la lengua y la brecha que puede existir entre los niveles de competencia curricular de los diferentes sistemas educativos de procedencia en comparación con los sistemas de acogida (Bleakley \& Chin, 2008; Corak, 2012; Fernández Enguita et al., 2010).

Finalmente, como última línea de investigación, nos planteamos analizar la discrepancia entre las exigencias curriculares entre los propios sistemas de acogida y los sistemas educativos de procedencia, porque se puede hipotetizar cuánto mayores serán la probabilidad de riesgo de bajo rendimiento y el posterior fracaso escolar.

\section{Aplicaciones en la práctica educativa}

Estos resultados nos llevan a plantear la importancia que tienen los planes de acogida en las primeras etapas del sistema educativo. Resulta necesario ser sensible a estas necesidades educativas, mantener una actitud proactiva ante el riesgo de fracaso escolar en etapas posteriores para prevenir y evitar el posterior abandono del sistema educativo por parte de escolares inmigrantes.

\section{Conflicto de interés}

La autora manifiesta que no posee conflicto de intereses.

\section{Agradecimientos}

Esteestudiofuefinalizadodurantelaestanciadeinvestigacióndesarrolladaenjuliode2016enelInstituto Nacional de Educación dependiente del Ministerio de Educación, Cultura y Deporte (Gobierno de España).

\section{Referencias}

Bleakley, H., \& Chin, A. (2008). What holds backs the second generation? Transmission of language human capital among immigrants. Journal of Human Resources, 43(2), 267-298.

Böhlmark, A. (2008). Age at immigration and school performance: A sibling's analysis using Swedish register data. Labour Economics, 15(6), 1366-1387. DOI:10.1016/j.labeco.2007.12.004

Bronfenbrenner,U.(1976). Theecology ofhumandevelopment:historyandperspectives.Psychologia, 19(5),537-549. 
E. BAusela

Calero, J. \& Escardíbul, J.O. (2013). El rendimiento del alumnado de origen inmigrante en PISA-2012. En MEC Y OCDE (2013). PISA 2012. Programa para la evaluación internacional de los alumnos informe español. Volumen II: Análisis secundario (pp. 5-31). Madrid: Ministerio de Educación, Cultura y Deporte.

Carabaña, J. (2012). Los efectos de la concentración de los inmigrantes sobre la composición social de las escuelas. Documento de Trabajo núm. 5, Sección de Sociología de la Educación, Universidad Complutense de Madrid: Madrid.

Cea D’Ancona, M.A.(2011). Análisis multivariable. Teoría y práctica en la investigación social. Madrid: Pirámide.

Cebolla-Boado, H. (2011). Primary and secondary effects in the explanation of immigrants' educational disadvantage. British Journal of Sociology, 32, 407-430. DOI:10.1080/01425692.2011.559341

Cebolla-Boado, H. (2012). La incorporación escolar de la población de origen inmigrante y el impacto de la concentración de inmigrantes en las escuelas navarras. Navarra: Departamento de Políticas Sociales. Sección de Atención a la Inmigración. Gobierno de Navarra: Comunidad Foral de Navarra.

Cebolla-Boado, H., \& Salazar, L. (2016). Differences in perinatal health among immigrant and native-origin children: evidence from differentials in birth weight in Spain. Demographic Research 35(7), 167-200. DOI: $10.4054 /$ DemRes.2016.35.7

Comunidad de Madrid. (s.f.). Plan PROA (Programa de Refuerzo Orientación y Apoyo) . Recuperado de http:// www.madrid.org/dat norte/plan_proa.htm

Corak, M. (2012). Age at inmigration and the education outcomes of children. En A. Masten, K. Liebkind y D.J. Hernandez (Eds.), Realizing the potential of immigration youth (pp. 90-116). Cambridge: Cambridge University Press.

Dustmann, C., Frattini, T., \& Lanzara, G. (2012). Educational achievement of second-generation immigrants: an international comparison. Economic Policy, 27(69), 143-185. DOI: http://dx.doi. $\operatorname{org} / 10.1111 / j .1468-0327.2011 .00275 . x$

Fernández Enguita, M., Mena, L. y Riviére, J. (2010). Fracaso y abandono escolar en España. Colección Estudios Sociales, $\mathrm{N}^{\circ} 29$. Barcelona: Fundación La Caixa.

Gould, E.D., Lavy, V., \& Paserman, M.D. (2009). Does immigration affect the long term educational outcomes of natives? Quasi - experimental evidence. The Economic Journal, 119, 1243-1269. DOI: 10.1111/j.1468-0297.2009.02271.x.

Heat, A., \& Brinbaum, Y. (2007). Explaining ethnic inequalities in educational attainment. Ethnicities 7 (3), 291-305. DOI: 35657522-398a-4c1a-8282-fee585066c3a.

Ley Orgánica 8/2013, de 9 de diciembre, para la mejora de la calidad educativa. Publicado en Boletín Oficial del Estado (BOE) $N^{\circ} 295$, del 10 de diciembre de 2013. España.

Ministerio de Educación, Cultura y Deporte (MECD) \& Organización para la Cooperación y el Desarrollo Económicos (OCDE) (Eds.) (2013a). PISA 2012, Programa para la Evaluación Internacional de los Alumnos. Informe Español, Volumen I: Resultados y contexto. Madrid: MEC- OECD. 
PISA 2012: InMigración y ETAPA DE LLEGAdA AL Sistema Educativo Español

Ministerio de Educación, Cultura y Deporte (MECD) \& Organización para la Cooperación y el Desarrollo Económicos (OCDE) (Eds.) (2013b). Marcos y pruebas de evaluación de PISA 2012. Madrid: MEC- OECD.

Ministerio de Educación, Cultura y Deporte (s.f.a). Educación Infantil. Recuperado de http://www.mecd.gob.es/ educacion-mecd/areas-educacion/estudiantes/educacion-infantil.html

Ministerio de Educación, Cultura y Deporte (s.f.b). Educación Primaria. Recuperado de http://www.mecd.gob. es/educacion-mecd/areas-educacion/estudiantes/educacion-primaria.html

Ministerio de Educación, Cultura y Deporte (s.f.c). Educación Secundaria. Recuperado de http://www.mecd. gob.es/educacion-mecd/areas-educacion/estudiantes/educacion-secundaria.html

Ministerio de Educación, Cultura y Deporte (s.f.d). Bachillerato. Recuperado de http://www.mecd.gob.es/ educacion-mecd/areas-educacion/estudiantes/bachillerato.html

Organisation for Economic Co-operation and Development (OECD) (2015). Can the performance gap between immigrant and non - immigrant students be closed? PISA IN FOCUS, 7. París: OECD Publishing.

Oliden, P.E(2013). Diferencias individuales y autonómicas en el estatus socioeconómico y cultural como predictores en PISA 2009. Revista de Educación, 361, 646-664. DOI:10.4438/1988-592X-RE-2013-361-236

Ozel, M., Caglak, S., \& Erdogan, M. (2013). Are affective factors a good predictor of science achievement? Examining the role of affective factors based on PISA 2006. Learning and Individual Differences 24, 73-82. DOI: 10.1016/j.lindif.2012.09.006

Pereda, C., de Prada M. A., \& Actis W. (1999). La inmigración extranjera en España, 2000. En E. A. Fernández (Coord.), La inmigración extranjera en España: los retos educativos (13-68). Colección Estudios Sociales, núm 1. Barcelona: Fundación La Caixa.

Rahona, M. \& Morales, S. (2013). Educación e inmigración en España: Desafíos y oportunidades. Series reformas educativas. Madrid: OEI con el apoyo de la Fundación Alternativas.

Real Decreto 1630/2006, de 29 de diciembre, por el que se establecen las enseñanzas mínimas del segundo ciclo de Educación Infantil (BOE de 4 de enero de 2007).

Real Decreto 126/2014, de 28 de febrero, por el que se establece el currículo básico de la Educación Primaria (BOE de 1 de marzo de 2014).

Real Decreto 1105/2014, de 26 de diciembre, por el que se establece el currículo básico de la Educación Secundaria Obligatoria y del Bachillerato (BOE de 3 de enero de 2015).

Schaafsma, J., \& Sweetman, A. (2001). Immigrant earnings: age at immigration matters. The Canadian Journal of Economics, 34(4), 1066-1099. DOI:10.1111/0008-4085.00113

Stiefl, L., Schwartz, A.E., \& Conger, D. (2010). Age of entry and the high school performance of immigrant youth. Journal of Urban Economics, 67, 303-314. DOI:10.1016/j.jue.2009.10.001

Van Ours, J.C., \& Veenman, J. (2006). Age at immigration and educational attainment of young immigrants. Economics Letters, 90, 310-316. DOI:10.1016/j.econlet.2005.08.013 
E. Bausela

Recibido: 31 de Enero de 2016

Revisión recibida: 29 de Marzo de 2017

Aceptado: 27 de Mayo de 2016

Sobre la autora:

Esperanza Bausela Herreras, es doctora cum laude en Psicología y Ciencias de la Educación por la Universidad de León y Especialista en Investigación Social Aplicada y Análisis de Datos por el Centro de Investigaciones Sociológicas. Ha desempeñado su actividad docente e investigadora en la Universidad Nacional de Educación a Distancia y, actualmente, en la Universidad Pública de Navarra. Ha sido investigadora visitante en la Universidad Autónoma de San Luis Potosí. Fue galardonada con el premio de Psicología Mar Mari y con el accésit del premio Mariano Rodríguez joven investigadora de la Fundación Carolina Rodríguez.

Publicado en línea: 26 de junio de 2017 
PISA 2012: InMigración y ETAPA DE LLEGAdA AL Sistema EduCATIVo EsPañol

\section{Anexos}

Anexo 1

Distribución de escolares inmigrantes en los distintos centros educativos en función de la edad de llegada al sistema educativo español

\begin{tabular}{|c|c|c|c|c|c|c|c|c|c|c|c|c|c|c|c|c|c|c|}
\hline \multirow{2}{*}{$\begin{array}{l}\text { Centros } \\
\text { educativos }\end{array}$} & \multicolumn{17}{|c|}{ Edad de llegada al sistema educativo español } & \multirow[t]{2}{*}{ Total } \\
\hline & 0 & 1 & 2 & 3 & 4 & 5 & 6 & 7 & 8 & 9 & 10 & 11 & 12 & 13 & 14 & 15 & 16 & \\
\hline 307 & 0 & 0 & 1 & 0 & 0 & 0 & 0 & 1 & 2 & 0 & 2 & 2 & 5 & 4 & 6 & 1 & 0 & 24 \\
\hline 160 & 0 & 0 & 0 & 0 & 1 & 4 & 0 & 1 & 1 & 0 & 2 & 3 & 4 & 4 & 0 & 1 & 0 & 21 \\
\hline 834 & 0 & 0 & 0 & 1 & 0 & 0 & 0 & 1 & 1 & 3 & 3 & 1 & 5 & 2 & 2 & 1 & 0 & 20 \\
\hline 77 & 0 & 0 & 2 & 2 & 2 & 1 & 4 & 0 & 1 & 2 & 1 & 1 & 2 & 0 & 0 & 0 & 0 & 18 \\
\hline 666 & 0 & 0 & 2 & 0 & 2 & 1 & 0 & 0 & 1 & 1 & 1 & 3 & 1 & 1 & 3 & 1 & 0 & 17 \\
\hline 533 & 0 & 0 & 0 & 2 & 2 & 0 & 3 & 0 & 2 & 2 & 1 & 0 & 0 & 0 & 2 & 2 & 0 & 16 \\
\hline 744 & 0 & 0 & 0 & 0 & 1 & 1 & 4 & 2 & 2 & 1 & 1 & 0 & 0 & 2 & 1 & 0 & 0 & 15 \\
\hline 47 & 1 & 0 & 0 & 1 & 1 & 0 & 2 & 2 & 0 & 3 & 4 & 0 & 0 & 0 & 0 & 0 & 0 & 14 \\
\hline 285 & 1 & 0 & 0 & 3 & 0 & 3 & 1 & 1 & 1 & 1 & 2 & 0 & 1 & 0 & 0 & 0 & 0 & 14 \\
\hline 523 & 0 & 0 & 0 & 1 & 0 & 1 & 0 & 0 & 0 & 3 & 1 & 3 & 1 & 1 & 0 & 3 & 0 & 14 \\
\hline 797 & 0 & 0 & 0 & 1 & 1 & 1 & 2 & 0 & 2 & 4 & 0 & 0 & 0 & 2 & 1 & 0 & 0 & 14 \\
\hline 19 & 0 & 0 & 0 & 0 & 1 & 1 & 0 & 0 & 2 & 1 & 4 & 2 & 1 & 0 & 1 & 0 & 0 & 13 \\
\hline 220 & 0 & 0 & 0 & 1 & 2 & 1 & 1 & 1 & 0 & 1 & 3 & 2 & 1 & 0 & 0 & 0 & 0 & 13 \\
\hline 412 & 0 & 0 & 0 & 2 & 3 & 4 & 1 & 0 & 0 & 2 & 0 & 0 & 1 & 0 & 0 & 0 & 0 & 13 \\
\hline 622 & 0 & 1 & 0 & 0 & 2 & 2 & 0 & 3 & 1 & 2 & 0 & 0 & 2 & 0 & 0 & 0 & 0 & 13 \\
\hline 638 & 0 & 0 & 0 & 0 & 0 & 2 & 0 & 1 & 0 & 3 & 0 & 3 & 2 & 1 & 1 & 0 & 0 & 13 \\
\hline 48 & 0 & 0 & 1 & 1 & 1 & 2 & 1 & 1 & 0 & 3 & 0 & 1 & 1 & 0 & 0 & 0 & 0 & 12 \\
\hline 113 & 0 & 0 & 1 & 0 & 2 & 1 & 2 & 0 & 1 & 0 & 0 & 3 & 2 & 0 & 0 & 0 & 0 & 12 \\
\hline 138 & 1 & 0 & 1 & 1 & 1 & 0 & 2 & 0 & 3 & 2 & 0 & 0 & 0 & 0 & 1 & 0 & 0 & 12 \\
\hline 193 & 0 & 0 & 0 & 0 & 1 & 2 & 4 & 1 & 1 & 2 & 1 & 0 & 0 & 0 & 0 & 0 & 0 & 12 \\
\hline 338 & 0 & 0 & 0 & 1 & 1 & 3 & 2 & 1 & 1 & 1 & 0 & 1 & 0 & 0 & 1 & 0 & 0 & 12 \\
\hline 393 & 0 & 0 & 1 & 2 & 2 & 2 & 3 & 1 & 0 & 0 & 0 & 0 & 1 & 0 & 0 & 0 & 0 & 12 \\
\hline
\end{tabular}

Continúa... 
E. Bausela

\begin{tabular}{|c|c|c|c|c|c|c|c|c|c|c|c|c|c|c|c|c|c|c|}
\hline 471 & 0 & 1 & 0 & 0 & 2 & 1 & 2 & 0 & 0 & 2 & 2 & 2 & 0 & 0 & 0 & 0 & 0 & 12 \\
\hline 614 & 0 & 0 & 0 & 0 & 1 & 1 & 5 & 1 & 1 & 0 & 2 & 0 & 1 & 0 & 0 & 0 & 0 & 12 \\
\hline 674 & 0 & 0 & 0 & 1 & 0 & 0 & 2 & 1 & 0 & 3 & 1 & 1 & 2 & 1 & 0 & 0 & 0 & 12 \\
\hline 126 & 0 & 0 & 0 & 0 & 3 & 0 & 0 & 2 & 0 & 1 & 1 & 2 & 0 & 1 & 1 & 0 & 0 & 11 \\
\hline 316 & 0 & 0 & 0 & 2 & 4 & 0 & 1 & 2 & 1 & 1 & 0 & 0 & 0 & 0 & 0 & 0 & 0 & 11 \\
\hline 407 & 0 & 0 & 0 & 1 & 0 & 4 & 1 & 0 & 0 & 2 & 0 & 2 & 0 & 0 & 0 & 1 & 0 & 11 \\
\hline 437 & 0 & 0 & 0 & 0 & 1 & 1 & 1 & 1 & 2 & 3 & 0 & 1 & 1 & 0 & 0 & 0 & 0 & 11 \\
\hline 440 & 0 & 0 & 0 & 0 & 1 & 1 & 0 & 1 & 0 & 1 & 4 & 1 & 0 & 1 & 1 & 0 & 0 & 11 \\
\hline 525 & 1 & 0 & 0 & 0 & 1 & 0 & 0 & 2 & 2 & 0 & 0 & 1 & 2 & 2 & 0 & 0 & 0 & 11 \\
\hline 803 & 1 & 0 & 0 & 0 & 0 & 0 & 1 & 1 & 3 & 0 & 1 & 1 & 1 & 0 & 0 & 1 & 1 & 11 \\
\hline 132 & 0 & 0 & 1 & 1 & 1 & 1 & 1 & 1 & 1 & 1 & 0 & 0 & 0 & 0 & 1 & 1 & 0 & 10 \\
\hline 137 & 0 & 0 & 1 & 0 & 0 & 0 & 2 & 3 & 0 & 0 & 3 & 0 & 0 & 1 & 0 & 0 & 0 & 10 \\
\hline 317 & 0 & 0 & 1 & 0 & 3 & 0 & 2 & 0 & 0 & 0 & 0 & 0 & 0 & 1 & 2 & 1 & 0 & 10 \\
\hline 398 & 0 & 0 & 0 & 0 & 1 & 3 & 0 & 1 & 0 & 1 & 0 & 3 & 0 & 1 & 0 & 0 & 0 & 10 \\
\hline 518 & 0 & 0 & 0 & 1 & 2 & 1 & 2 & 2 & 0 & 0 & 1 & 0 & 1 & 0 & 0 & 0 & 0 & 10 \\
\hline 524 & 0 & 0 & 1 & 0 & 0 & 1 & 0 & 1 & 1 & 1 & 1 & 1 & 2 & 1 & 0 & 0 & 0 & 10 \\
\hline 546 & 1 & 0 & 1 & 0 & 0 & 0 & 0 & 1 & 0 & 2 & 1 & 2 & 2 & 0 & 0 & 0 & 0 & 10 \\
\hline 547 & 0 & 0 & 1 & 0 & 1 & 0 & 1 & 1 & 1 & 1 & 1 & 1 & 2 & 0 & 0 & 0 & 0 & 10 \\
\hline 767 & 1 & 0 & 0 & 0 & 1 & 0 & 1 & 1 & 1 & 1 & 1 & 1 & 1 & 0 & 1 & 0 & 0 & 10 \\
\hline Otros & 57 & 52 & 109 & 124 & 167 & 223 & 190 & 185 & 161 & 146 & 210 & 169 & 142 & 86 & 87 & 41 & 8 & 2157 \\
\hline Total & 64 & 54 & 124 & 149 & 215 & 272 & 244 & 224 & 197 & 202 & 253 & 215 & 191 & 115 & 111 & 55 & 9 & 2694 \\
\hline
\end{tabular}


PISA 2012: InMigración y ETAPA DE LLEGAdA AL Sistema EduCATIVo EsPañol

Anexo 2

Distribución de escolares inmigrantes en los distintos centros educativos en función de la etapa educativa de llegada al sistema educativo español

\begin{tabular}{|c|c|c|c|c|c|}
\hline \multirow[b]{2}{*}{ Centros educativos } & \multicolumn{4}{|c|}{ Etapa educativa de llegada al sistema educativo español } & \multirow[b]{2}{*}{ Total } \\
\hline & Educación infantil & $\begin{array}{l}\text { Educación } \\
\text { primaria }\end{array}$ & $\begin{array}{c}\text { Educación secundaria } \\
\text { obligatoria }\end{array}$ & Bachillerato & \\
\hline 307 & 1 & 7 & 16 & 0 & 24 \\
\hline 160 & 5 & 7 & 9 & 0 & 21 \\
\hline 834 & 1 & 9 & 10 & 0 & 20 \\
\hline 77 & 7 & 9 & 2 & 0 & 18 \\
\hline 666 & 5 & 6 & 6 & 0 & 17 \\
\hline 533 & 4 & 8 & 4 & 0 & 16 \\
\hline 744 & 2 & 10 & 3 & 0 & 15 \\
\hline 47 & 3 & 11 & 0 & 0 & 14 \\
\hline 285 & 7 & 6 & 1 & 0 & 14 \\
\hline 523 & 2 & 7 & 5 & 0 & 14 \\
\hline 797 & 3 & 8 & 3 & 0 & 14 \\
\hline 19 & 2 & 9 & 2 & 0 & 13 \\
\hline 220 & 4 & 8 & 1 & 0 & 13 \\
\hline 412 & 9 & 3 & 1 & 0 & 13 \\
\hline 622 & 5 & 6 & 2 & 0 & 13 \\
\hline 638 & 2 & 7 & 4 & 0 & 13 \\
\hline 48 & 5 & 6 & 1 & 0 & 12 \\
\hline 113 & 4 & 6 & 2 & 0 & 12 \\
\hline 138 & 4 & 7 & 1 & 0 & 12 \\
\hline 193 & 3 & 9 & 0 & 0 & 12 \\
\hline 338 & 5 & 6 & 1 & 0 & 12 \\
\hline 393 & 7 & 4 & 1 & 0 & 12 \\
\hline 471 & 4 & 8 & 0 & 0 & 12 \\
\hline 614 & 2 & 9 & 1 & 0 & 12 \\
\hline
\end{tabular}

Continúa... 
E. Bausela

\begin{tabular}{|c|c|c|c|c|c|}
\hline 674 & 1 & 8 & 3 & 0 & 12 \\
\hline 126 & 3 & 6 & 2 & 0 & 11 \\
\hline 316 & 6 & 5 & 0 & 0 & 11 \\
\hline 407 & 5 & 5 & 1 & 0 & 11 \\
\hline 437 & 2 & 8 & 1 & 0 & 11 \\
\hline 440 & 2 & 7 & 2 & 0 & 11 \\
\hline 525 & 2 & 5 & 4 & 0 & 11 \\
\hline 803 & 1 & 7 & 2 & 1 & 11 \\
\hline 132 & 4 & 4 & 2 & 0 & 10 \\
\hline 137 & 1 & 8 & 1 & 0 & 10 \\
\hline 317 & 4 & 2 & 4 & 0 & 10 \\
\hline 398 & 4 & 5 & 1 & 0 & 10 \\
\hline 518 & 4 & 5 & 1 & 0 & 10 \\
\hline 524 & 2 & 5 & 3 & 0 & 10 \\
\hline 546 & 2 & 6 & 2 & 0 & 10 \\
\hline 547 & 2 & 6 & 2 & 0 & 10 \\
\hline 767 & 2 & 6 & 2 & 0 & 10 \\
\hline Otros & 143 & 274 & 109 & 1 & 527 \\
\hline Total & 878 & 1335 & 472 & 9 & 2694 \\
\hline
\end{tabular}

